\title{
Identificación de Factores de Riesgo de la Diabetes Mellitus Tipo 2 en Adultos de 30 a 60 Años de edad en la Comunidad de Isla Aguada, Municipio de Ciudad del Carmen, Campeche.
} Identifying Risk Factors for Type 2 Diabetes Mellitus in Adults 30 to 60 years of age in the Community of Isla Aguada, municipality of Ciudad del Carmen, Campeche.

Betty Sarabia Alcocer Universidad Autónoma de Campeche, México bmsarabi@uacam.mx

Ana Rosa Can Valle Universidad Autónoma de Campeche, México anarocan@uacam.mx

Jaqueline Guadalupe Guerrero Ceh Universidad Autónoma de Campeche, México jgguerre@uacam.mx

\section{Resumen}

La Diabetes Mellitus es un síndrome metabólico caracterizado por hiperglucemia, consecuencia de defectos en la secreción y/o en la acción de la insulina. La hiperglucemia crónica se asocia en largo plazo con daño, disfunción e insuficiencia de diferentes órganos especialmente de los ojos, riñones, nervios, corazón y vasos sanguíneos.

Objetivo: Identificar los factores de riesgo de Diabetes Mellitus de tipo 2 en una población rural del estado de Campeche. Material y Métodos; Se realizó un estudio observacional, de tipo descriptivo, transversal y retrospectivo, a 213 individuos, de entre 30 y 60 años de edad, adscritos al Centro de Salud de Isla Aguada, Campeche, con diagnóstico de Diabetes Mellitus de tipo 2 ,en periodo 2010 a 2011. Se registró los datos de sexo, edad, antecedentes heredo-familiares, índice de masa corporal y valores de colesterol y 
triglicéridos. Resultados: Se encontró que la edad promedio en la que se presenta la Diabetes Mellitus de tipo 2 es de 50.64, con predominio en mujeres, hay un incremento en el riesgo directamente proporcional a la edad; la obesidad representa el $61.5 \%$, la dislipidemia un $41.3 \%$ y los antecedentes heredo-familiares el $36 \%$ de riesgo. Conclusiones: Los factores de riesgo relacionados a Diabetes Mellitus de tipo 2, en la población de Isla Aguada, fueron principalmente la obesidad y la dislipidemia, encontrándose predominio en mujeres y un aumento del riesgo a padecerla proporcional a la edad.

Palabras claves: Factores de Riesgo, Diabetes Mellitus, adultos.

\section{Abstract}

Diabetes mellitus is a metabolic syndrome characterized by hyperglycemia resulting from defects in secretion and / or insulin action. (1) Chronic hyperglycemia is associated in longterm damage, dysfunction and failure of various organs, especially the eyes, kidneys, nerves, heart and blood vessels.

Objective: To identify risk factors for Type 2 Diabetes Mellitus in a rural population of the state of Campeche. Material and methods; An observational study of 213 individuals, aged between 30 and 60 years of age, assigned to the Health Center of Isla Aguada, Campeche, diagnosed with Type 2 Diabetes Mellitus was performed, descriptive, crosssectional and retrospective, in period 2010 to 2011. Data on sex, age, family history-I inherited, BMI and cholesterol and triglyceride values were recorded. Results: We found that the average age at which diabetes mellitus type 2 is 50.64 occurs, predominantly women, there is an increased risk directly proportional to age; Obesity accounts for $61.5 \%$, 41.3\% dyslipidemia and history-family inherited 36\% risk. Conclusions: The risk factors related to type 2 Diabetes Mellitus, in the town of Isla Aguada, were mainly obesity and dyslipidemia, being more common in women and increased risk to suffer proportional to age.

Key words: Risk Factors, Diabetes Mellitus, adults. 
Fecha Recepción: Junio 2015 Fecha Aceptación: Noviembre 2015

\section{Introducción}

La Diabetes Mellitus de tipo 2 es una enfermedad crónica de gran interés epidemiológico, debido a que el efecto del progreso en la sociedad, ha tenido gran relación con el aumento de la prevalencia e incidencia de ésta enfermedad, en otras palabras, la Diabetes Mellitus de tipo 2, ha ido en incremento paralelo con la obesidad, el sedentarismo y la edad. La Organización Mundial de la Salud (OMS) ha pronosticado que en el año 2030 esta enfermedad afectará a 370 millones de personas, lo que supone un aumento de un 114\% a nivel mundial con respecto al año 2000; se estima que el continente americano ocupa el tercer lugar en incidencia de esta enfermedad, y que en México se sitúa como la $7^{\mathrm{a}}$ causa de muerte.

En estudios previos se ha encontrado una fuerte asociación de la obesidad, el antecedente familiar de Diabetes Mellitus de tipo 2, la edad, la ingestión de grasas saturadas y la dislipidemia, entre otros, con la aparición de diabetes mellitus de tipo 2.

Tomando en cuenta los datos estadísticos y que cada vez se diagnostica más diabetes Mellitus de tipo 2, y casi siempre es en estadios avanzados de complicaciones tardías, se decide la realización de este estudio como un reto para el diagnóstico temprano de ésta enfermedad, a través de un amplio conocimiento de los factores de riesgo asociados, presentes en la comunidad de Isla Aguada a fin de proponer una estrategia preventiva de la enfermedad.

El conocimiento de los factores de riesgo específicos en la comunidad permitirá desarrollar actividades preventivas, promotoras, y políticas de salud a fin de modificarlos en la población de mayor riesgo y de ese modo disminuir las tasas de la enfermedad y de sus complicaciones.

El objetivo principal del presente estudio fue conocer la frecuencia de los factores de riesgo asociados a diabetes Mellitus de tipo 2, en la población de Isla Aguada. 


\section{MATERIAL Y METODOS}

Se realizó un estudio observacional, de tipo descriptivo, transversal y retrospectivo, en la comunidad de Isla Aguada, municipio Ciudad del Carmen, Campeche; se llevó a cabo entre el periodo de 01 de Agosto de 2010 al 31 de Julio de 2014, mediante la selección de los expedientes de sujetos mayores, de entre 30 y 60 años de edad, adscritos al centro de Salud de la comunidad de Isla Aguada, con Diagnostico de Diabetes Mellitus de Tipo 2.

Criterios de Inclusión: Personas de ambos sexos, de entre 30 y 60 años de edad, con diagnóstico de Diabetes Mellitus de tipo 2, que cuenten con expediente clínico en el Centro de Salud de Isla Aguada. Independientemente de pertenecer o no al programa de Seguro Popular y que sea habitante de la localidad.

Criterios de Exclusión: Pacientes con expediente clínico incompleto, con patologías múltiples o que no se incluyan dentro de la Diabetes Mellitus de tipo 2.

Se seleccionaron únicamente los expedientes que cumplían de manera estricta con estos criterios, obteniendo un tamaño de la muestra de 213 expedientes, de los cuales se obtuvieron datos correspondientes al sexo, edad, antecedentes heredo-familiares, obesidad y valores bioquímicos de colesterol y triglicéridos actualizados (resultados de un lapso no mayor a 6 meses). Posteriormente, ésta información se recolecto de manera individual en las hojas de registro.

Procedimiento estadístico: Los datos fueron resumidos en una hoja de cálculo y procesados. Los resultados (medidas de tendencia central y números absolutos), se representaron como apoyo, a manera de gráficos. 


\section{RESULTADOS}

Las características de la población,

Se trata de una muestra de 213 individuos con rango de edad entre 30 y 60 años, de ambos sexos, 96 hombres y 117 mujeres, todos ellos pobladores de Isla Aguada con diagnóstico de Diabetes Mellitus de tipo 2. Durante el estudio, el promedio de edad para presentar la Diabetes Mellitus de tipo 2, es de 50.64 años (Tabla 1)

\section{TABLA 1.}

Características de la Población Estudiada $(n=213)$

\begin{tabular}{lll}
\hline & Rango & Promedio \\
\hline Sexo & $\begin{array}{c}\text { Masculino /femenino } \\
96 / 117\end{array}$ & \\
& & \\
Edad & 30 a 60 años & 50.64 \\
\hline
\end{tabular}

Clasificado por rango de edades encontramos que hay una mayor frecuencia en individuos de entre 46 a 60 años de edad, que resultó en un total de 124 casos, representando un $58.2 \%$, comparado con el $41.8 \%$ de prevalencia, en individuos de entre 30 a 45 años, con un total de 89 casos. (Tabla 2) (Gráfico 1). 


\section{TABLA 2}

Prevalencia por rango de edades
EDAD
N
PORCENTAJE

(AÑOS)

$30-45$

89

$41.8 \%$

$46-60$

124

$58.2 \%$

\section{GRÁFICO 1}

\section{Prevalencia por Rango de edades}

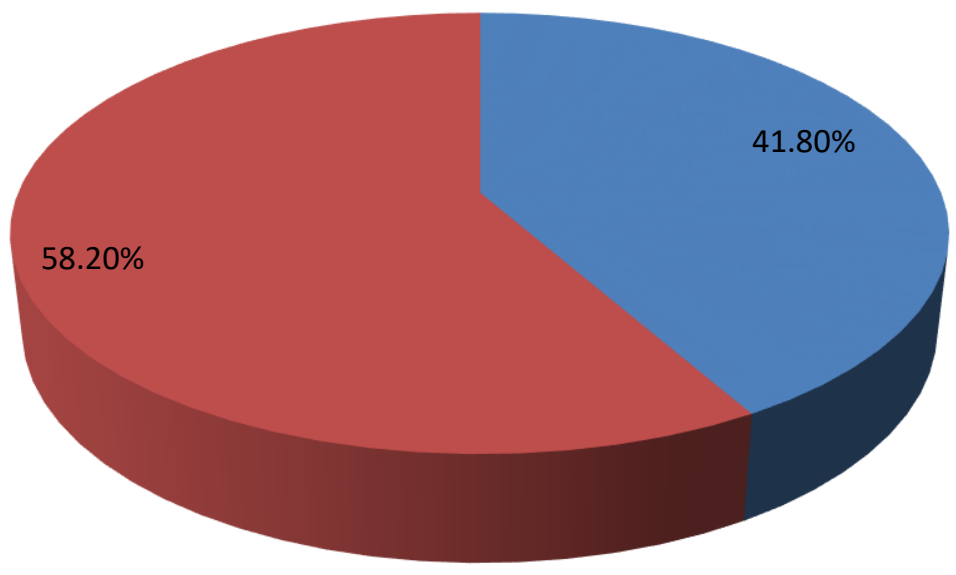

- 30 a 45 años

- 46 a 60 años

Referente al sexo, como ya se mencionó, se estudiaron un total de 213 individuos, 96 de ellos hombres, portadores de DM de tipo 2 y 117 mujeres (Tabla 3) con el mismo diagnóstico, por lo que se encontramos una tendencia con predominio en el género femenino con un 55\%, comparado con el $45 \%$ de prevalencia en hombres. (Gráfico 2) 


\section{TABLA 3}

Prevalencia de Diabetes Mellitus de tipo 2 Asociada al sexo

\section{GRÁFICO 2}

\section{Prevalencia de DM2 asociada al sexo}
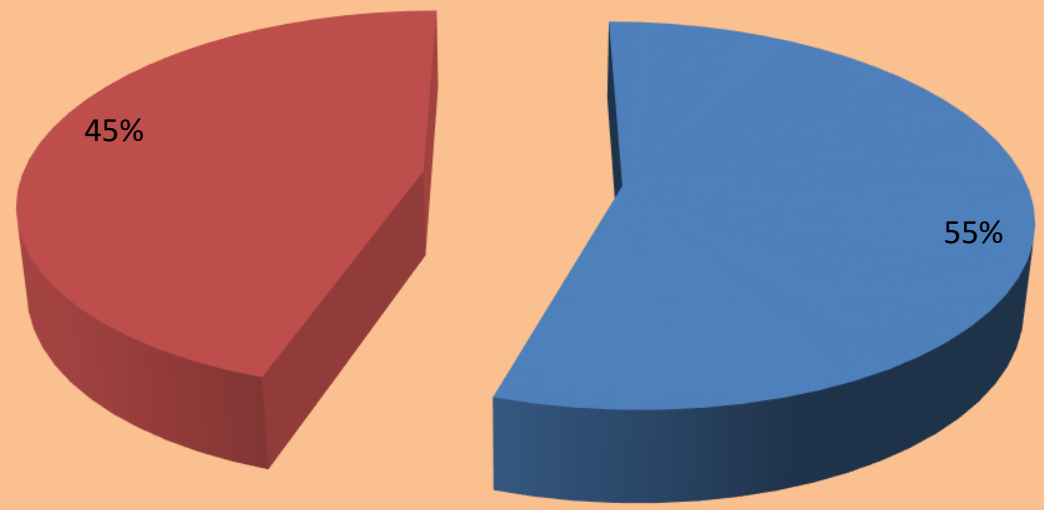

mujer

nombre

El 36\% de la población tiene antecedentes de DM de tipo 2 por línea directa, ya sea en abuelos, padres o hermanos, con un total de 77 casos. Se encontró una prevalencia de $61.5 \%$ de obesidad, 131 casos, con índice de masa corporal mayor de 30, Se halló dislipidemia en un 41.3\%, con un total de 88 casos (Tabla 4) (Gráfico 3). 


\section{TABLA 4}

Porcentajes de la muestra que reportaron condición de riesgo

ANTECEDENTES

HEREDO-FAMILIARES

OBESIDAD

\section{GRAFICO 3}

\section{PORCENTAJE DE RIESGO DE LOS CASOS}

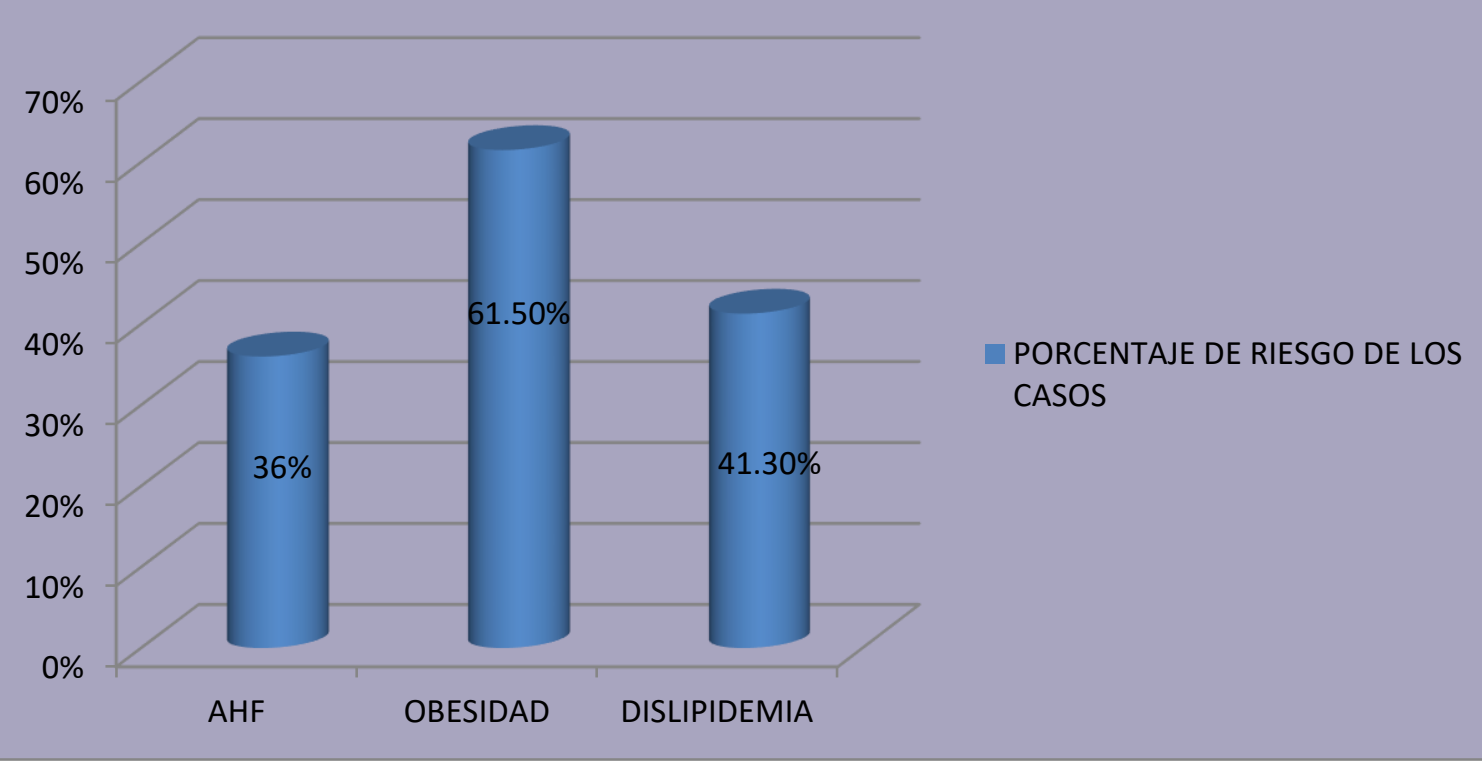


Se halló dislipidemia en un $41.3 \%$, con un total de 88 casos, de los cuales el $19.24 \%$, es decir 41 pacientes, presentaron hipercolesterolemia mayor de $200 \mathrm{mg} / \mathrm{dL}$ y un $22.06 \%, 47$ pacientes, con hipertrigliceridemia mayor de 150mg/dL. (Tabla 5 ) (Gráfico 4) (Gráfico 5)

\section{TABLA 5}

Dislipidemia como factor de Riesgo

\begin{tabular}{lcc}
\hline TIPO & N & PORCENTAJE \\
\hline HIPERCOLESTEROLEMIA & 41 & $19.2 \%$ \\
HIPERTRIGLICERIDEMIA & 47 & $22.1 \%$ \\
\hline \hline TOTAL & 88 & $41.3 \%$ \\
\hline
\end{tabular}

\section{GRÁFICO 4}

\section{PORCENTAJE DE CASOS CON DISLIPIDEMIA COMO FACTOR DE RIESGO}

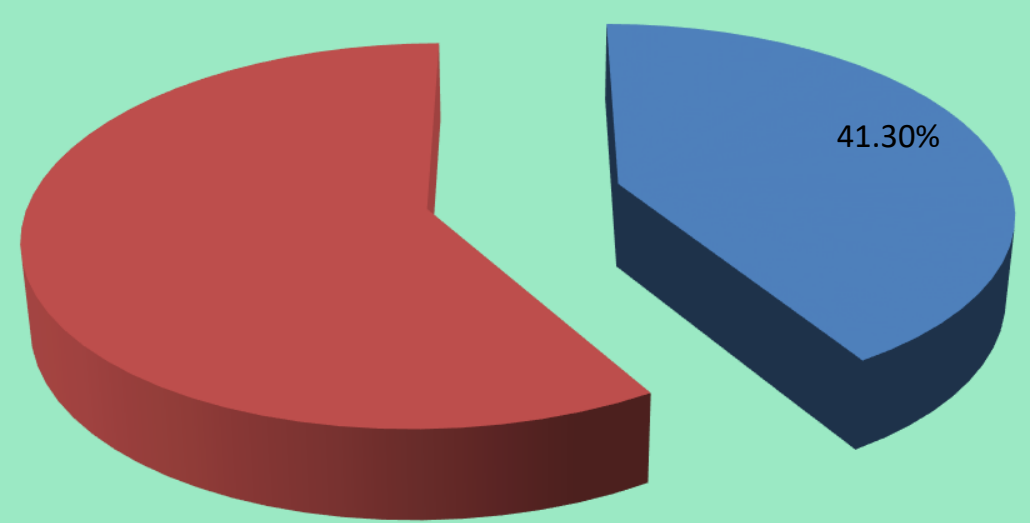

DISLIPIDEMIA

- SIN DISLIPIDEMIA 


\section{GRÁFICO 5}

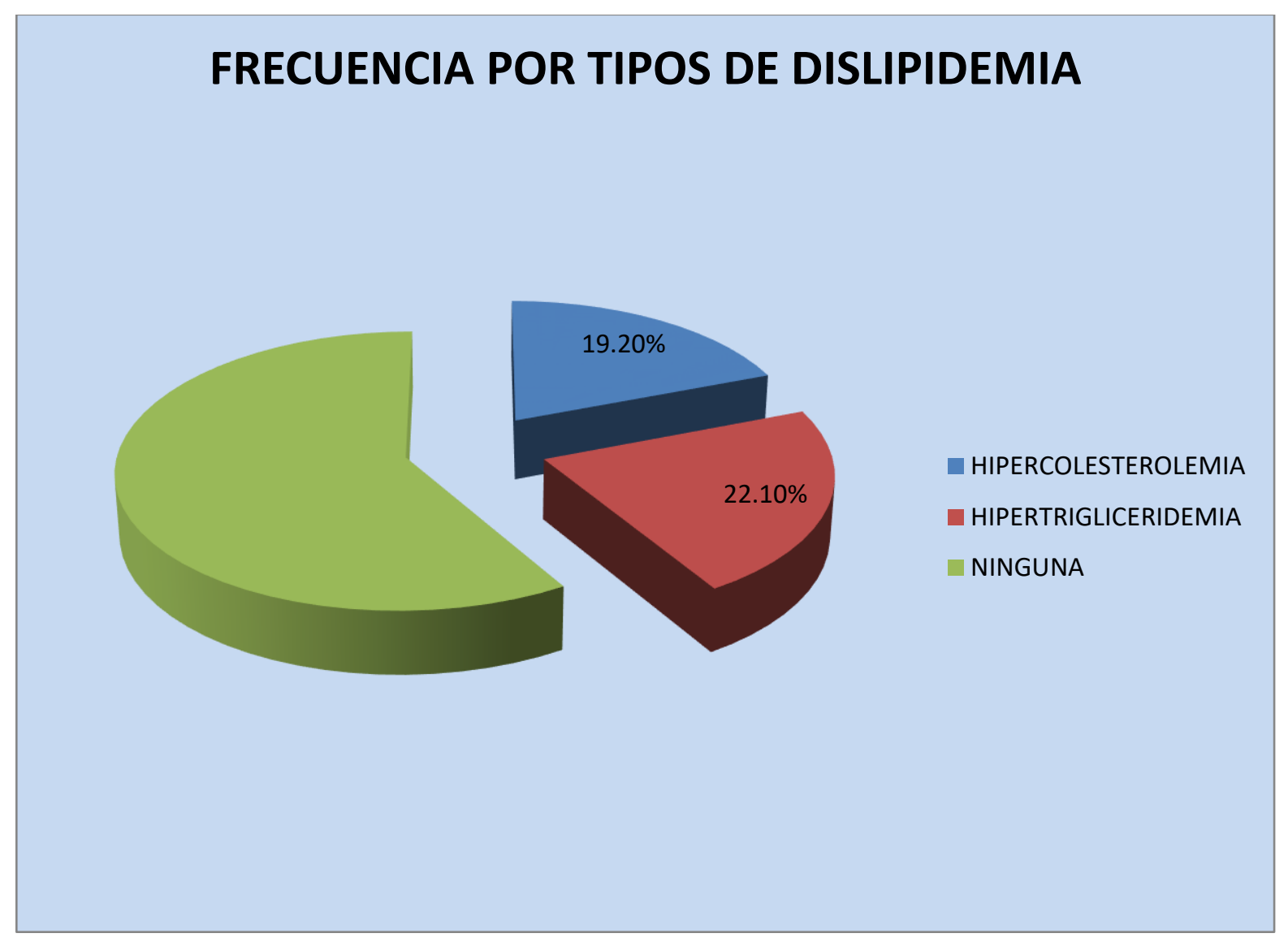

Dicho todo lo anterior encontramos un $36 \%$ de población con antecedentes heredofamiliares(n=77), un $61.5 \%$ de prevalencia de obesidad $(n=131)$ y un $41.3 \%$ de dislipidemia $(n=88)$, como factor de riesgo para diabetes mellitus de tipo 2. (Grafico 3) (Tabla 4)

\section{DISCUSIÓN}


En este estudio encontramos un $61.5 \%$ de hallazgo de obesidad de la población de Isla Aguada. Dichos valores fueron muy altos, comparados con los obtenidos en estudios realizados en población de Breña, Lima con resultados de $22.8 \%$, sin embargo hay que considerar el tipo de selección de los sujetos, los cuales además pertenecen a una población rural y las edades comprendidas son mayores de 15 años, lo cual hace que varíe de un estudio a otro, tomando en cuenta que el universo de la muestra y que la Diabetes Mellitus de tipo 2, esta asociada a un incremento acorde al aumento de edad, explicaría la elevada prevalencia de obesidad en nuestro estudio, aplicado a personas mayores, y los márgenes de diferencia tan amplios entre ambas investigaciones.

Es muy importante hacer mención que la OMS considera al Caribe como una región donde el aumento del número de diabéticos hasta 2025, será $150 \%$, además de que la mayoría, estaría entre 45 y 64 años y una relación hombre:mujer de 0:7; es decir mayor predominio femenino.

Estas proyecciones significan un incremento notable del número de diabéticos, los que demandarían, atención médica y sufrirían de las complicaciones tardías que suelen acompañar a estos pacientes en los países subdesarrollados. Así los costos estimados que la diabtes produce en cuanto a detección, manejo y prevención secundaria serían muy grandes.

En relación a los factores de riesgo estudiados y asociados a DM de tipo 2, también se encontró que hay una prevalencia significativa con relación a la herencia, tal como se esperada y se ha sustentado en diversos estudios, que abordan éste tema, realizados en México.

Otro un dato que llama la atención son las cifras de colesterol y triglicéridos con frecuencia en $19.24 \%$ y $22.06 \%$ respectivamente, relativamente bajas y con una considerable diferencia en otros estudios realizados, con cifras que duplican nuestros resultados, una posible explicación a este fenómeno, sea que difiere el estatus económico de las poblaciones de estudio, por lo que el tipo de alimentación también es diferente, En las poblaciones urbanas encontramos predominantemente una dieta de alto contenido calórico 
con excesiva ingesta de grasas saturadas y nuestra población de estudio es de un medio rural lo cual podrá explicar nuestros resultados.

El reconocer a la obesidad, la herencia, la edad, las dislipidemias, como factores de predisposición para Diabetes Melllitus de tipo 2,su manejo oportuno y adecuada educación del paciente de alto riesgo, sin duda alguna reducirá las cifras de prevalencia y morbimortalidad de este padecimiento, que cada día van en aumento en México y en nuestra población de estudio, he aquí la importancia de la realización del presente estudio.

\section{CONCLUSIONES}

- La obesidad ha demostrado ser el principal factor de riesgo para presentar Diabetes Mellitus de tipo 2, lo cual concuerda en la mayoría de los estudios realizados en México.

- Definitivamente la prevalencia de la enfermedad se incrementa conforme aumenta la edad.

- Los antecedentes heredo-familiares de diabetes Mellitus de tipo 2, en consanguíneos directos, ha demostrado ser un importante factor de riesgo para manifestar ésta enfermedad, lo cual apoya el carácter hereditario de este padecimiento.

- En la actualidad no existe ninguna tendencia congruente en la frecuencia de la enfermedad según el sexo, aún cuando los datos apuntan a un exceso d predisposición en la mujer, no es suficiente, por lo que ésta idea aún es imprecisa.

- La obesidad es un factor modificable, por lo cual su detección temprana, en pacientes con elevada predisposición de Diabetes Mellitus de tipo 2 (portador de más de 1 factor de riesgo) y eliminación de dicha condición, disminuirá significativamente la prevalencia de la enfermedad y la aparición de complicaciones tempranas como tardías. 


\section{Bibliografía}

Tao-Le, Vikas Bhushan, AArup Kubal, Jae Park., First AID para el Internado, $3^{\mathbf{a}}$ Ed., México, Mc Graw Hill, 2006, 156.

Arteaga A. Maiz A., Olmos P. y Velasco N. Manual de Diabetes y Enfermedades Metabólicas. Depto.Nutrición, Diabetes y Metabolismo. Escuela de Medicina. P. Universidad Católica de Chile. 1997

American Diabetes Association. Report of the Expert Committe on the Diagnosis and Clasification of Diabetes. Diabetes Care 1997; 20 (7): p.1183

Kasper D., Braunwald E., Fauci A., Hauser S., Longo D., Jameson J. Harrison Principios de Medicina Interna, 16a Ed, Mc Graw Hill, 2005, 2: p.2368

Manuel H., José Halabe, Alberto L., Joaquín López. El Internista, 2ª Ed., Mc Graw Hill, 2002 , p.40.

Fletcher, Gulanick \& Lamendola, C. Risk Factor for type 2 diabetes mellitus, The journal of cardiovascular nursing, 2002, 16 (2), 17-23.

Arauco O. Frecuencia de diabetes mellitus y factores de riesgo asociados en el distrito deVilla el Salvador- Lima. Tesis de Maestría, UPCH, Lima, 1999.

Tuomilehto J, Lindstrom J, Eriksson JG, Valle TT, Hamalainen H, Ilanne-Parikka P, Keinanen-Kiukaanniemi S, et al. Prevention of type 2 diabetes mellitus by changes in lifestyle among subjects with impaired glucose tolerance. N Engl J Med 2001; 344:1343-50.

World Health Organization (WHO). Physical Status: The use and interpretation of anthropometry. Report of WHO Expert Committee. Technical Report Series No.854. Geneva: World Health Organization; 1995.

Andreoli T., Bennett J., Carpenter Charles, Plum Fred., Cecil Compendio de Medicina Interna. $4^{\mathrm{a}}$ Ed,. México, Mc Graw Hill, 1999, p. 526.

Srinivasan SR., Myers L., Berenson GS., Rate of change in adiposity and its relationship to concomitant change in cardiovascular risk variables among biricial (black-white.) children and young adults: The Bugalusa Heart Study. Metabolism. 2001; 50: 299305.

Lerman G., Clasificación y Diagnostico de la Diabetes Mellitus, Atención Integral del paciente Diabético, México,Mc Graw Hill- Interamericana, 2003; 7-24. 
Ferrannini E., Natali A., Bell P., Cavallo-Perin P., Lalic N., and Mingrone G. on behalf of the European Group for the study of insulin Resistence (EGIR) et al. Insulin Resistance and Hypersecretion in Obesity. J Clin Invest 1997; 100:1166-1173.

Diabetes Prevention Program Research Group. Reduction in the incidence of Type 2 Diabetes with Lifestyle intervention or Metformin. N Engl J Med 2002; 346:393403.

Sacks D. \& MacDonald J: The pathogenesis of type 2 Diabetes Mellitus. AJCP 1996; 105: 149-57

Farreras V.,C. Rozman. , Medicina Interna. 15ª Ed., España; Elsevier, 2004; 2: 1948-1949

POTTER, Patricia A. Valoración Física. Guías para profesionales de enfermería. Ed. Interamericana. (Disponible en la Biblioteca "Graciela Arroyo de Cordero" de la ENEO)

Meigs JB. , Cupples LA, Wilson PW. Parental transmission of type 2 diabetes. The Framingham Offspring Study. Diabetes. 2000; 49(12) 2201-7

Barnett AH; Eff C., Leslie RD, Pyke DA. Diabetes in identical twins. A study of 200 pairs 50. Diabetologia.1981; 20(2):87-93.

Stephen J McPhee, Maxine A. Papadakis. , Diagostico Clínico y Tratamiento,49 Ed,México Mc Graw Hill 2010; 1081

Encuestas de Salud Pública en México, años 2000-2003

Age- And- Sex - Specific prevalences of diabetes and impaired glucose regulation in 13 European cohorts. Diabetes Care.2003; 26 (1) :69-9 http://www.asocimed.cl/Guias\%20Clinicas/endocrinologia/dislipidemias.html

Salud Pública en México 2007; 47:03-04: 2

Garmendia F. Prevención en diabetes mellitus. Diagnóstico 1998; 37(2):113-9. 


\section{HOJA DE RECOLECCIÓN DE DATOS}

DIAGNOSTICO: DIABETES MELLITUS TIPO 2

SEXO

NUMERO DE EXPEDIENTE

EDAD

ASPECTOS ANTOPOMÉTRICOS:

PESO

Kgs TALLA

$\mathrm{mts}$

IMC

OBESIDAD:

PRESENTE:

AUSENTE:

ANTECEDENTES HEREDO - FAMILIARES

\begin{tabular}{|c|l|}
\hline Familiares Consanguíneos $\geq 18$ años & Diagnóstico de Diabetes Mellitus 2 \\
\hline Abuelos & \\
\hline Padres & \\
\hline Hermanos & \\
\hline
\end{tabular}

"En caso positivo anotar quien y en caso negativo anotar "ninguno".

LABS: FECHA DE TOMA:

Colesterol Total $\mathrm{mg} / \mathrm{dl}$

Triglicéridos $\mathrm{mg} / \mathrm{dl}$

DISLIPIDEMIA:

PRESENTE:

AUSENTE:

Fecha 